Supplement of Atmos. Chem. Phys., 22, 371-393, 2022

https://doi.org/10.5194/acp-22-371-2022-supplement

(C) Author(s) 2022. CC BY 4.0 License.

(c) (1)

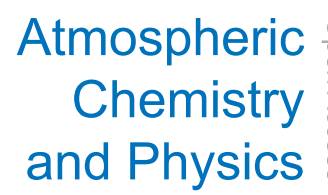

Supplement of

\title{
Exploration of the atmospheric chemistry of nitrous acid in a coastal city of southeastern China: results from measurements across four seasons
}

Baoye Hu et al.

Correspondence to: Jinsheng Chen (jschen@iue.ac.cn) and Min Qin (mqin@aiofm.ac.cn)

The copyright of individual parts of the supplement might differ from the article licence. 


\section{Supplementary Material}

Table S1. Comparison of HONO concentrations and related parameters at this site with other regions.

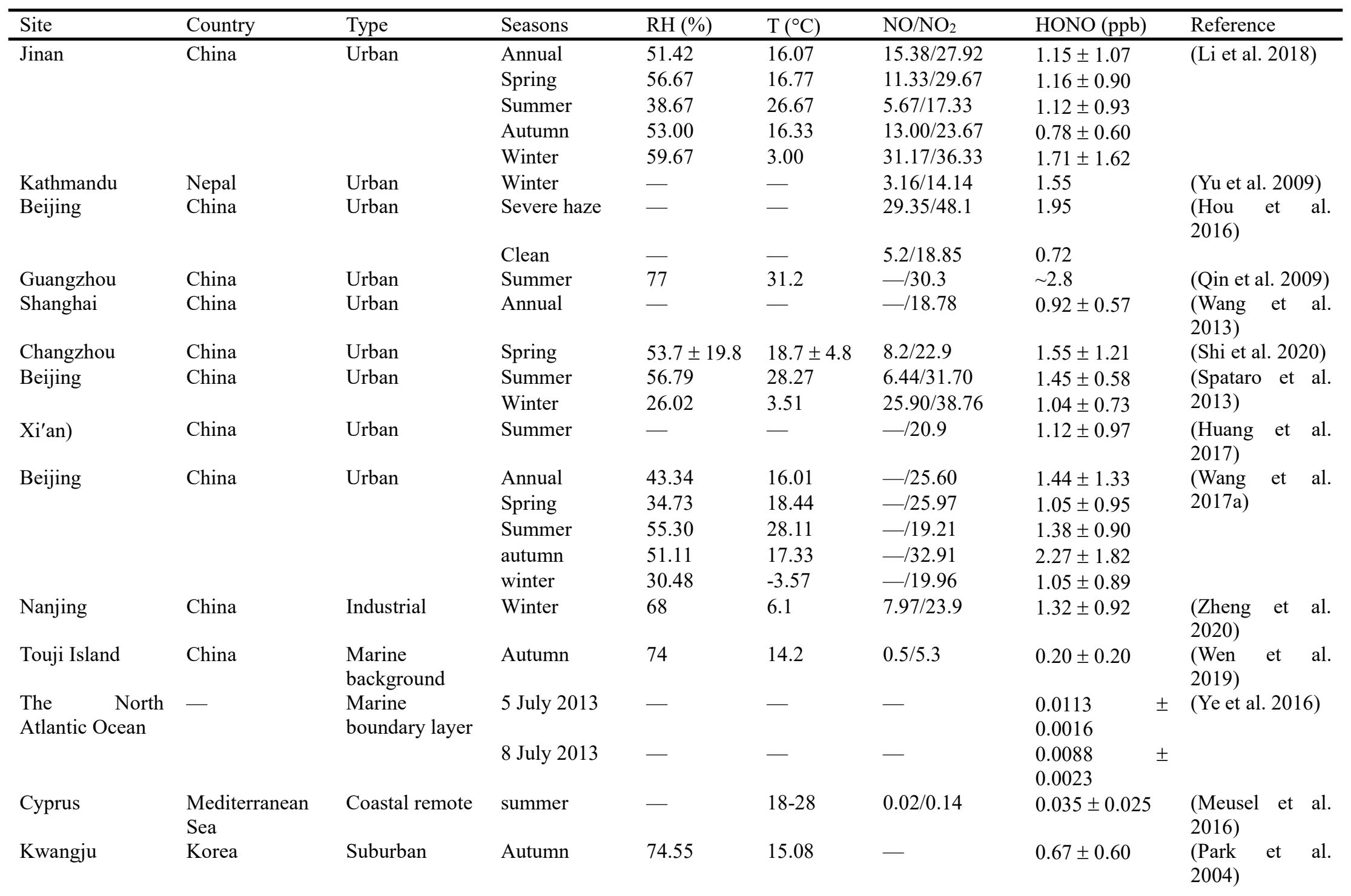




\begin{tabular}{|c|c|c|c|c|c|c|c|c|}
\hline \multirow[t]{5}{*}{ Nanjing } & \multirow[t]{5}{*}{ China } & \multirow[t]{5}{*}{ Suburban } & Annual & 72 & 17.00 & $5.7 / 16.4$ & $0.69 \pm 0.58$ & \multirow[t]{5}{*}{ (Liu et al. 2019) } \\
\hline & & & Spring & 73 & 17.67 & $2.35 / 15.15$ & $0.68 \pm 0.48$ & \\
\hline & & & Summer & 77 & 28.00 & $1.2 / 10.1$ & $0.45 \pm 0.37$ & \\
\hline & & & Autumn & 72 & 18.11 & $5.25 / 16.15$ & $0.66 \pm 0.53$ & \\
\hline & & & Winter & 66 & 4.33 & $15.58 / 25.75$ & $1.04 \pm 0.75$ & \\
\hline \multirow[t]{5}{*}{ Hongkong } & \multirow[t]{5}{*}{ China } & \multirow[t]{5}{*}{ Suburban } & Annual & 73 & 25 & $10.7 / 21.7$ & 0.71 & \multirow[t]{5}{*}{ (Xu et al. 2015) } \\
\hline & & & Spring & 75 & 28 & $5.5 / 15.5$ & 0.35 & \\
\hline & & & Summer & 71 & 32 & $8 / 19.8$ & 0.65 & \\
\hline & & & Autumn & 67 & 23 & $10.1 / 26.8$ & 0.93 & \\
\hline & & & Winter & 78 & 17 & $19.3 / 24.7$ & 0.91 & \\
\hline \multirow{6}{*}{$\begin{array}{l}\text { Western Yangtze } \\
\text { River delta } \\
\text { Xiamen }\end{array}$} & China & Suburban & Spring & - & - & - & $0.76 \pm 0.79$ & (Nie et al. 2015) \\
\hline & \multirow[t]{5}{*}{ China } & \multirow[t]{5}{*}{ Suburban } & Annual & 78.35 & 22.95 & $5.80 / 14.99$ & $0.54 \pm 0.47$ & \multirow[t]{5}{*}{ This work } \\
\hline & & & Spring & 84.21 & 16.59 & $8.47 / 18.10$ & $0.62 \pm 0.58$ & \\
\hline & & & Summer & 84.12 & 30.00 & $4.79 / 13.39$ & $0.61 \pm 0.39$ & \\
\hline & & & Autumn & 69.55 & 24.02 & $2.18 / 12.88$ & $0.41 \pm 0.30$ & \\
\hline & & & Winter & 78.13 & 18.41 & $8.86 / 17.03$ & $0.54 \pm 0.47$ & \\
\hline
\end{tabular}

Note: "_-" means no data found in the corresponding reference. 


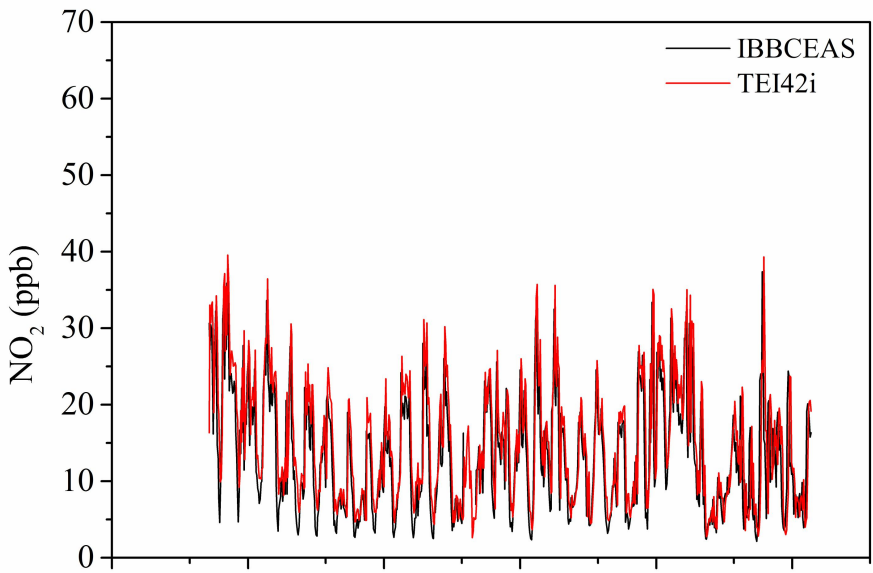

Jul $27 \quad$ Aug 3 Aug 10 Aug 17 Aug 24 Aug 31

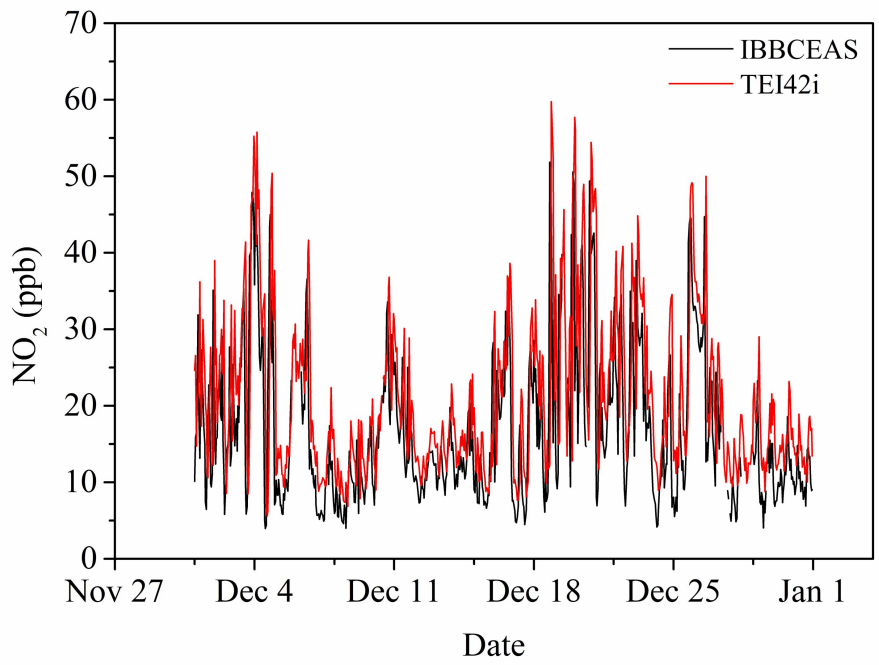

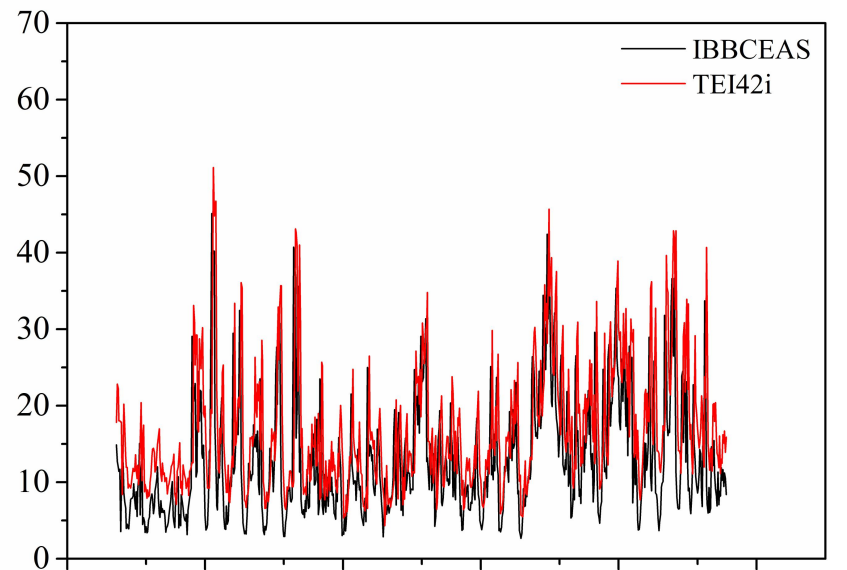

$\begin{array}{llllll}\text { Sep } 28 & \text { Oct } 5 & \text { Oct } 12 & \text { Oct } 19 & \text { Oct } 26 & \text { Nov } 2\end{array}$

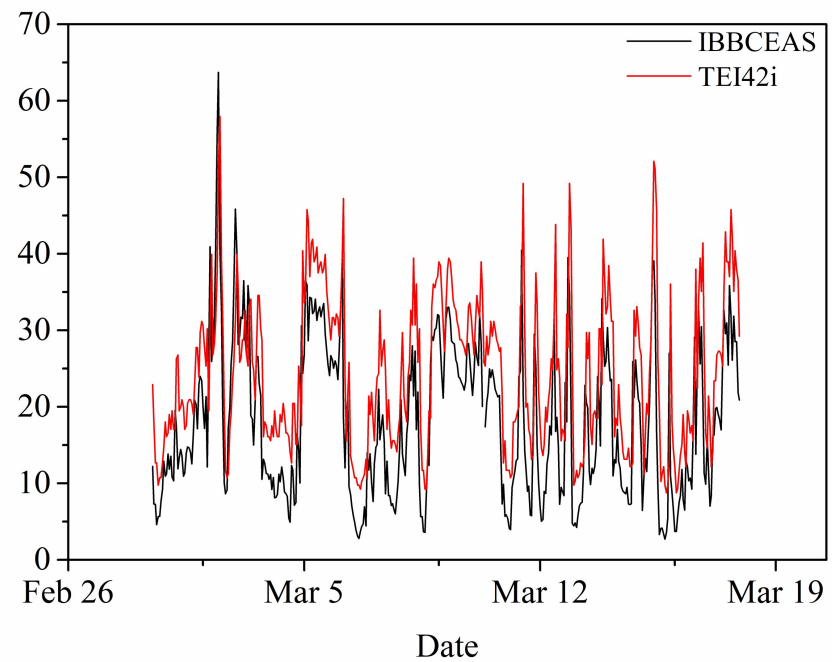

Figure S1. Time series measurements of $\mathrm{NO}_{2}$ from the IBBCEAS and TEI $42 i$. 


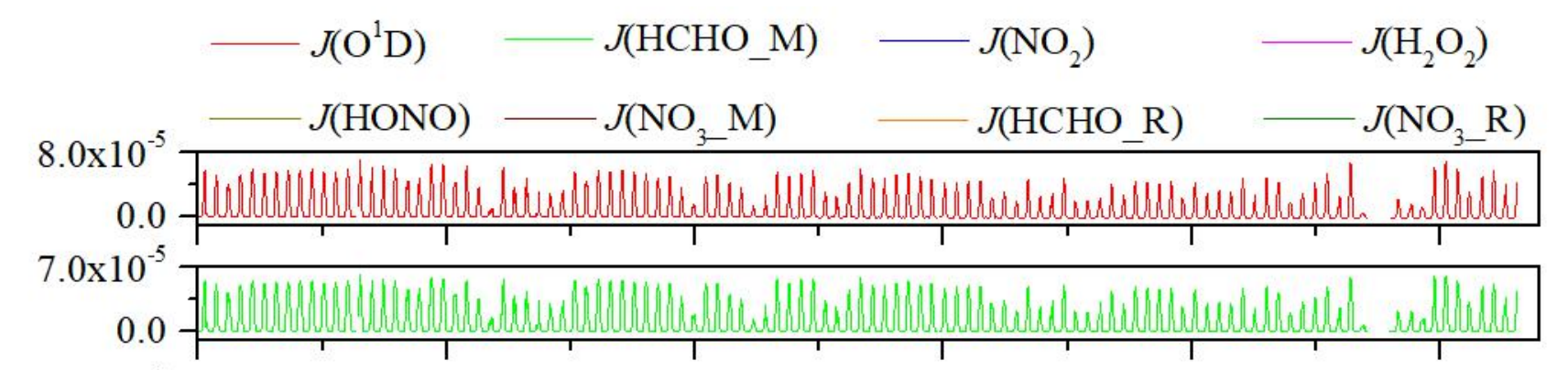

$1.30 \times 10^{-2}$

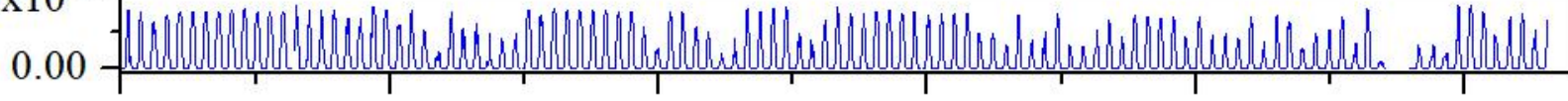

$1.40 \times 10^{-5}$

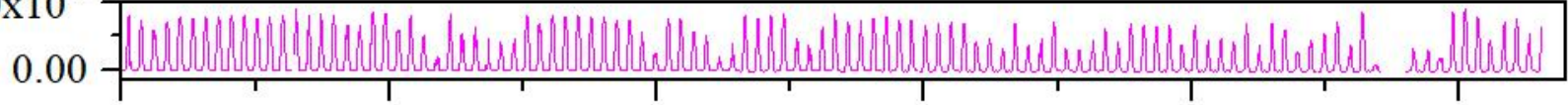

$2.250 \times 10^{-3}$

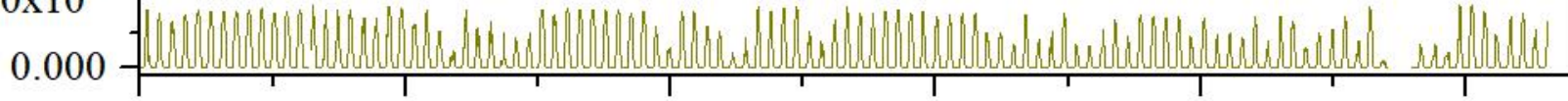

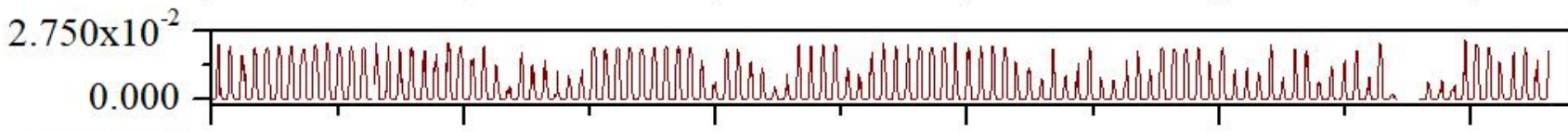

$6.50 \times 10^{-5}$

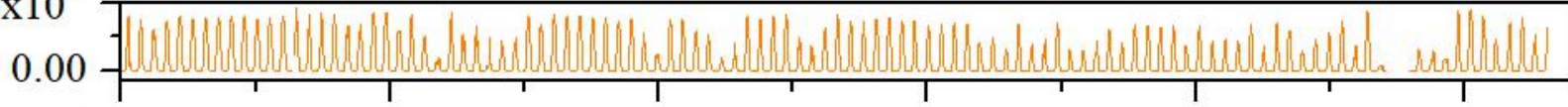

$2.250 \times 10^{-1}$

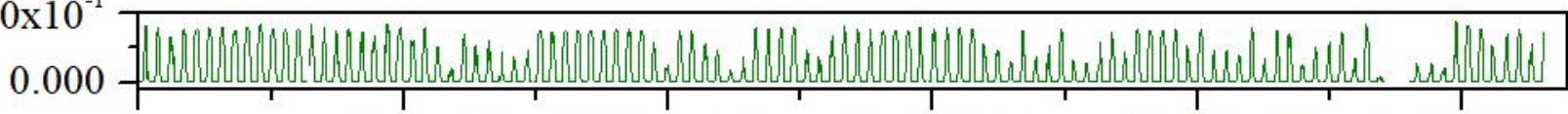
2018/8/21 2018/10/11 2018/12/1 2018/12/22 2019/3/12

Figure S2. Time series of photolysis rate constants. 


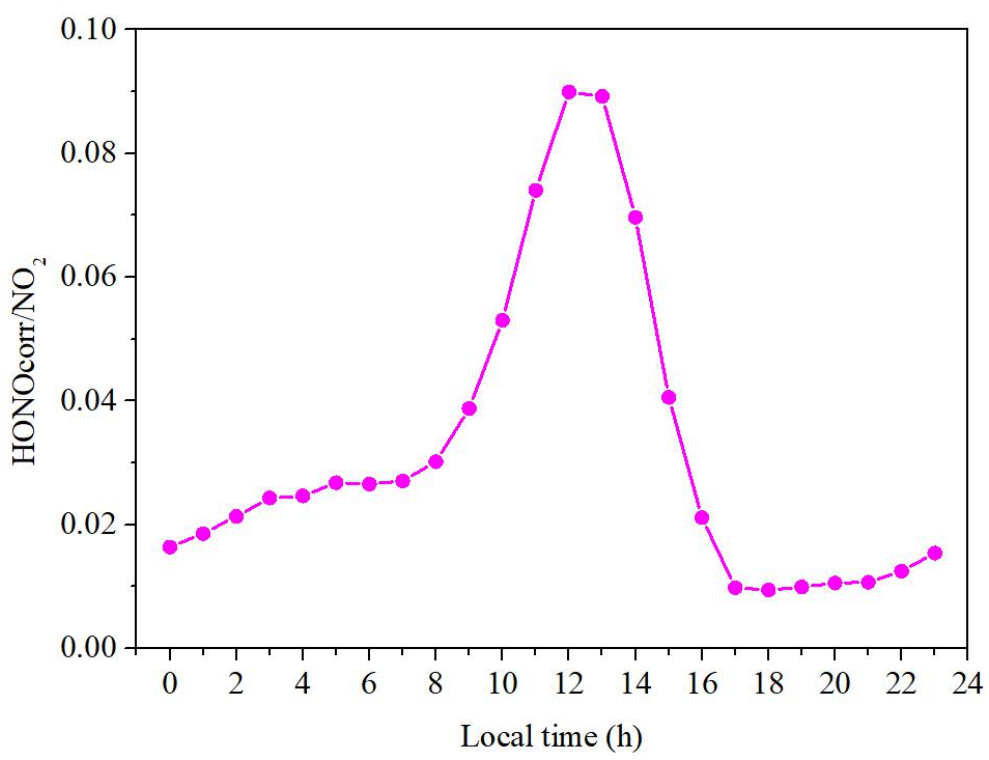

Figure S3. Diurnal variations of $\mathrm{HONO}_{\text {corr }} / \mathrm{NO}_{2}$. 

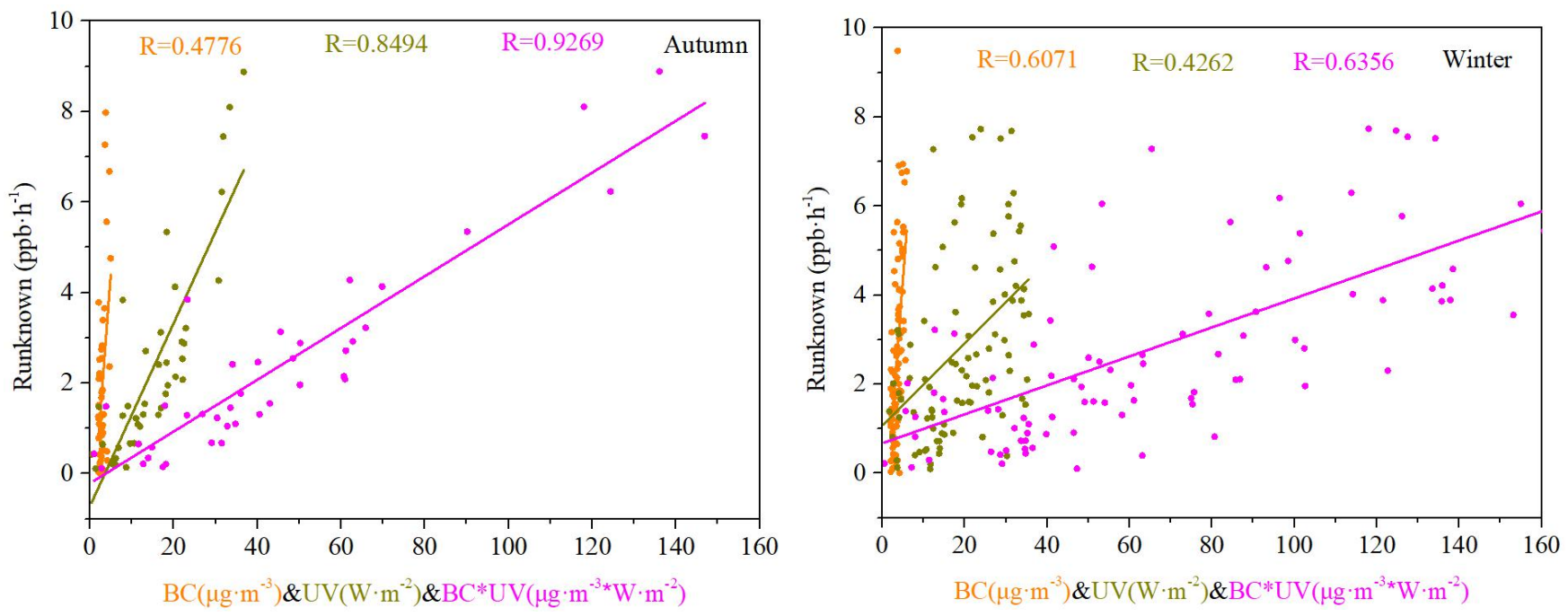

15 Figure S4. Correlations between the strength of Runknown and BC (colored by orange), UV (colored by dark yellow), and $\mathrm{BC} * \mathrm{UV}$ (colored by magenta) in autumn and winter, respectively 

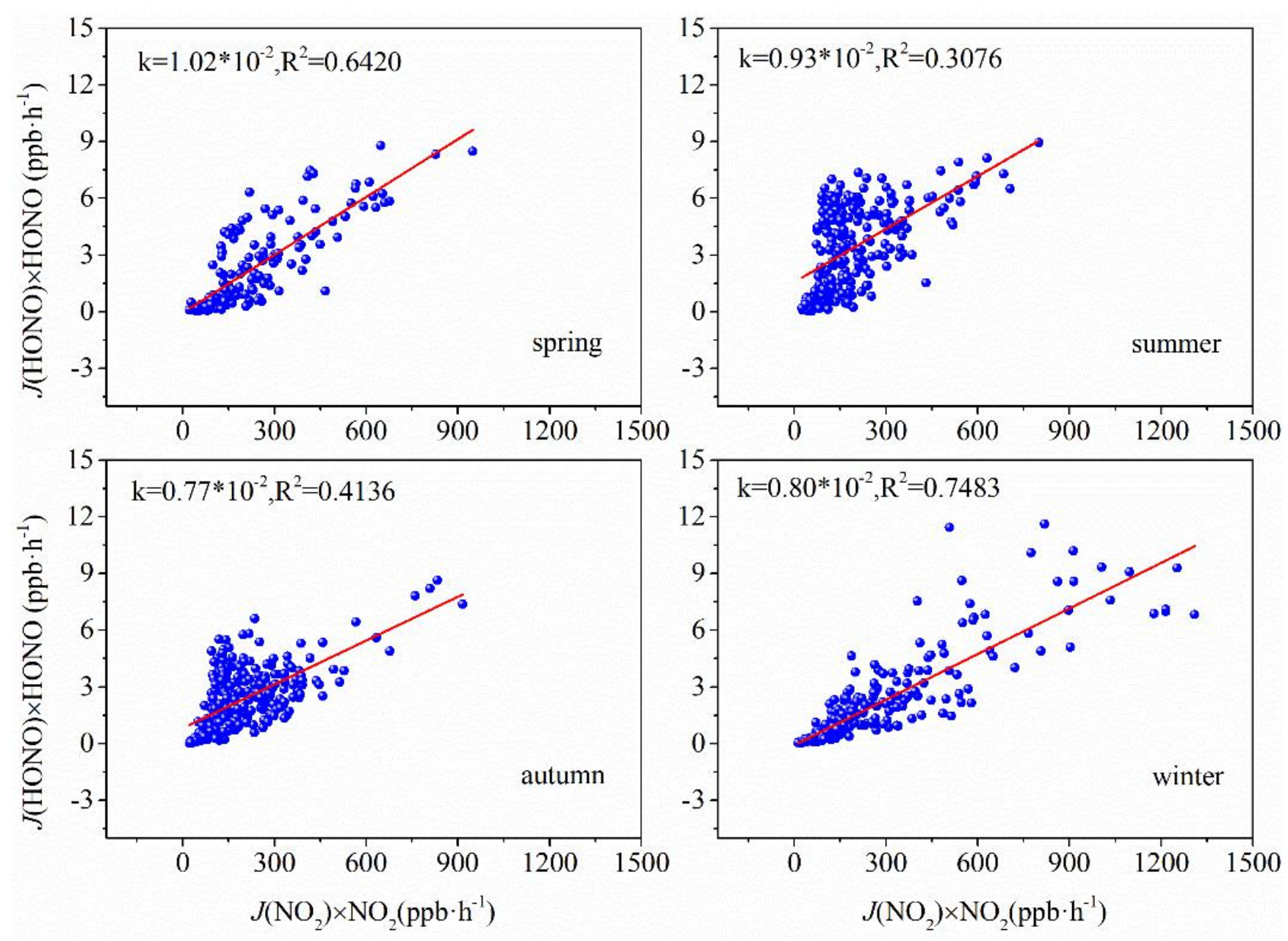

Figure S5. Correlations between $J(\mathrm{HONO}) \times \mathrm{HONO}$ and $J\left(\mathrm{NO}_{2}\right) \times \mathrm{NO}_{2}$. 
- HONOobs - HONOsim
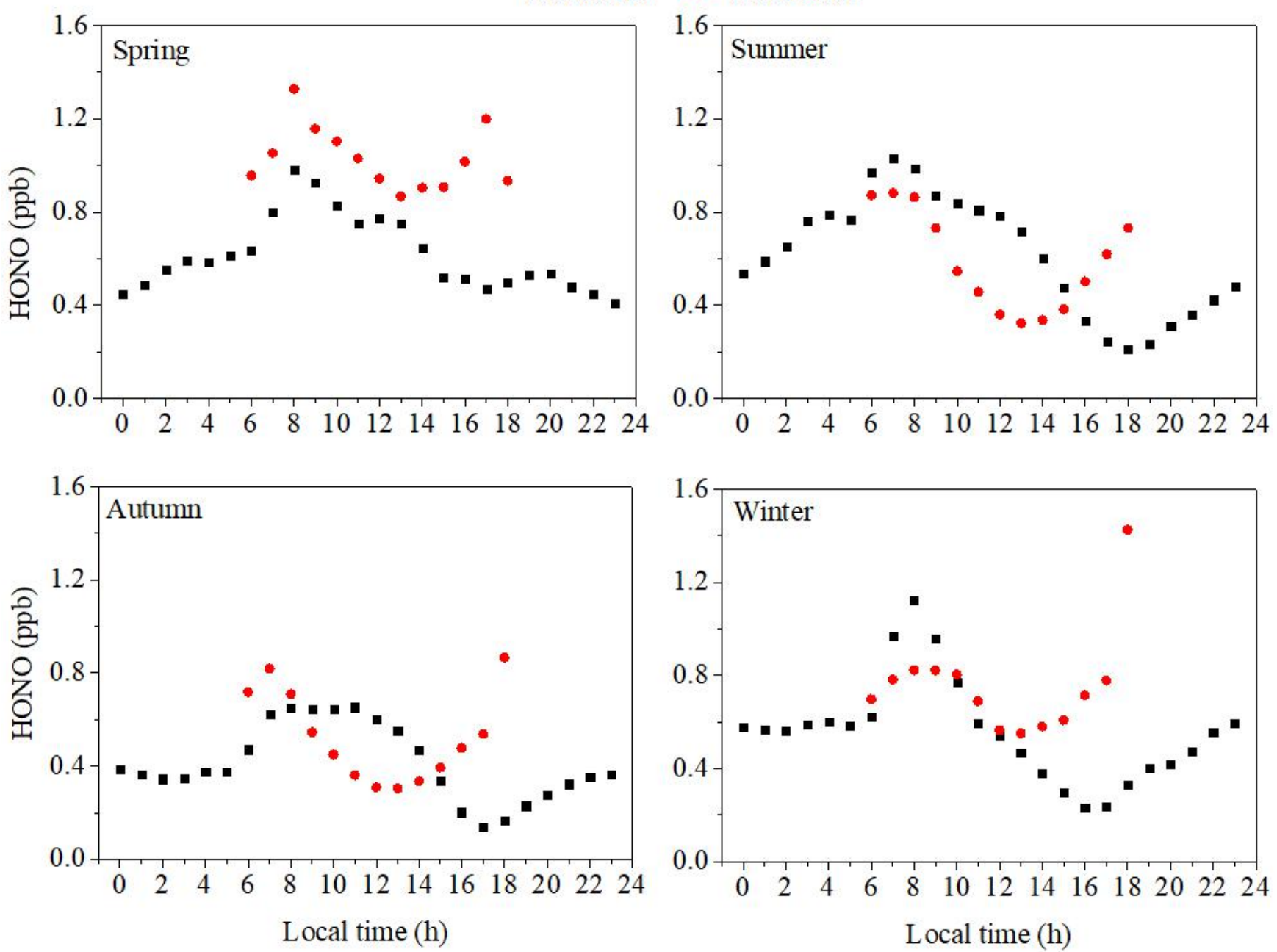

Figure S6. Diurnal profile of the measured values of HONO and the value estimated using the parameterized formula Eq. (10). 

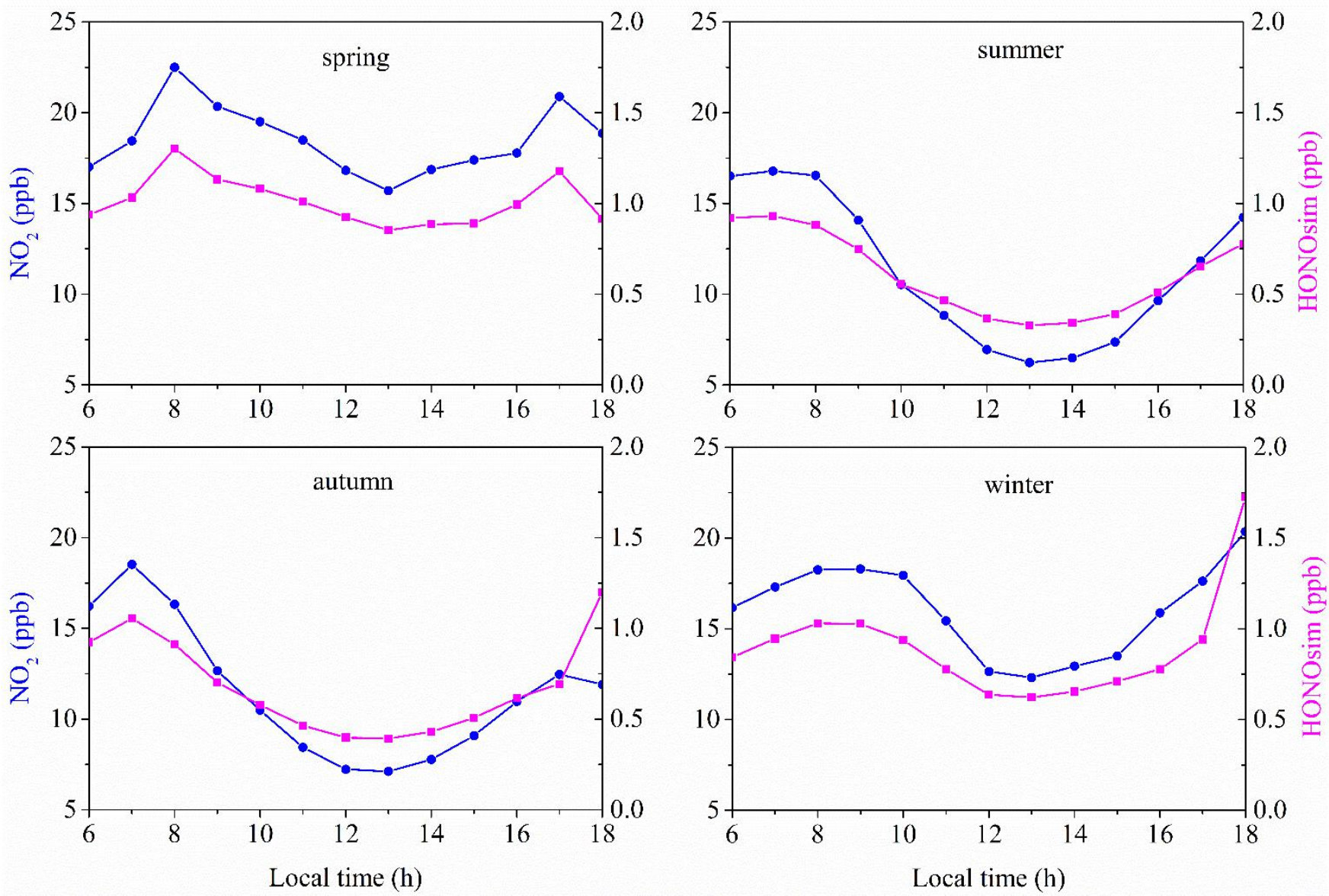

Figure S7. Diurnal variation of $\mathrm{NO}_{2}$ concentration and $\mathrm{HONO}$ concentration simulated by Eq. (10). 


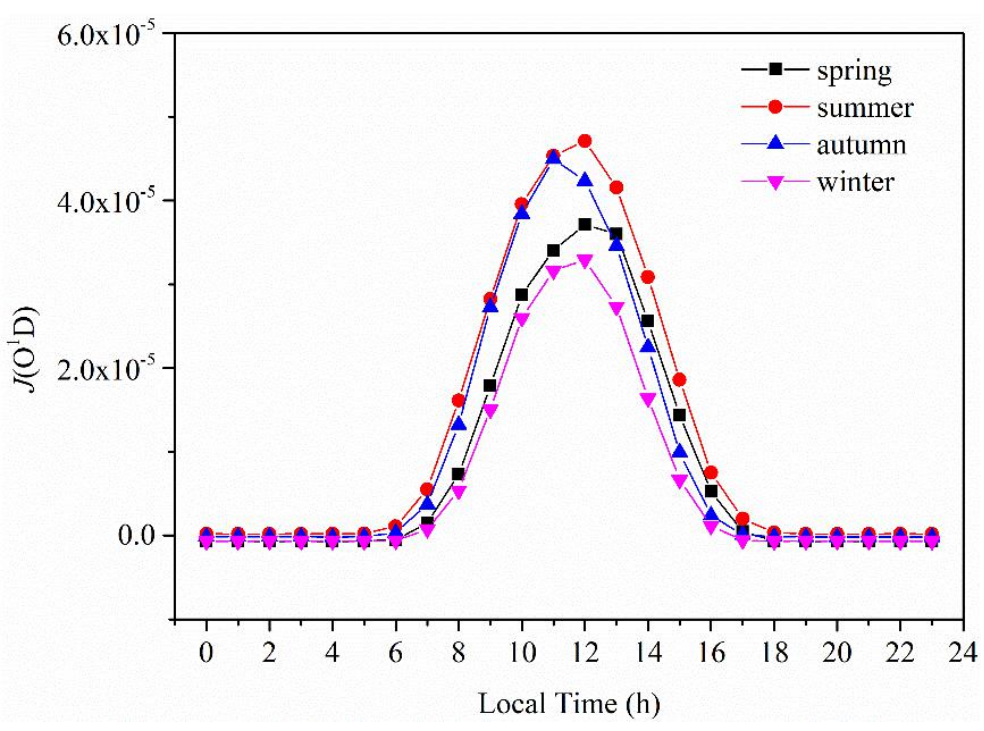

Figure S8. Diurnal variations of $J\left(\mathrm{O}^{1} \mathrm{D}\right)$. 


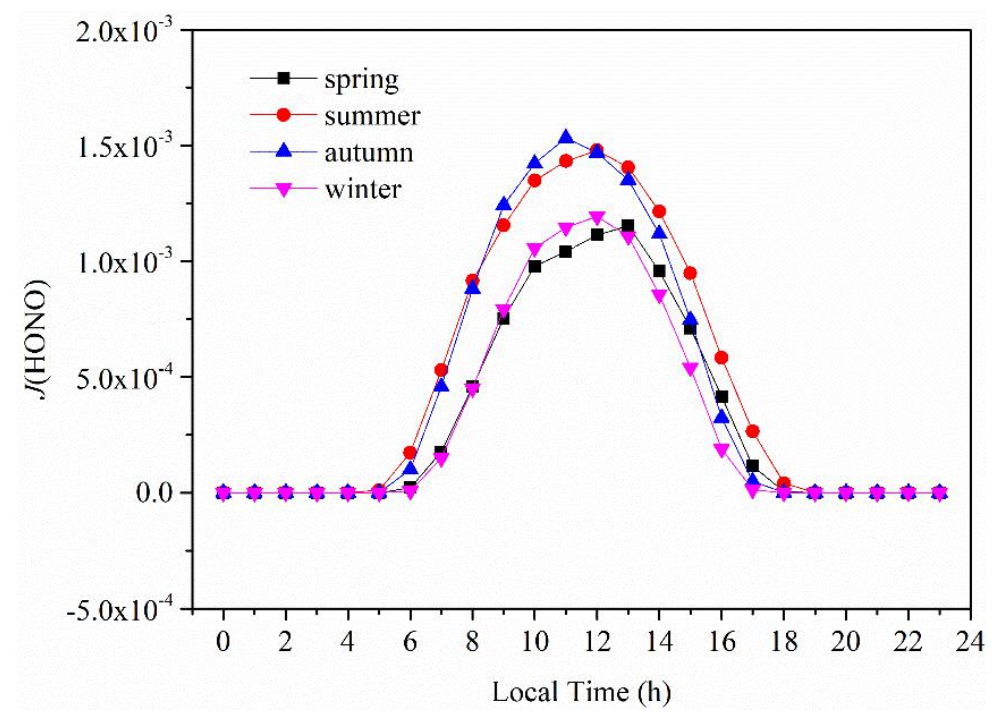

Figure S9. Diurnal variations of $J(\mathrm{HONO})$. 